\title{
Hurst Exponent Analysis on the Ghana Stock Exchange
}

\author{
Isaac Ampofi ${ }^{1}$, Akyene Tetteh ${ }^{2}$, Eric Neebo Wiah ${ }^{1}$, Sampson Takyi Appiah $^{3}$ \\ ${ }^{1}$ Mathematical Sciences Department, University of Mines and Technology, Tarkwa, Ghana \\ ${ }^{2}$ Management Studies Department, University of Mines and Technology, Tarkwa, Ghana \\ ${ }^{3}$ Mathematics and Statistics Department, University of Energy and Natural Resources, Sunyani, Ghana
}

\section{Email address:}

ampofiisaac34@gmail.com (I. Ampofi)

\section{To cite this article:}

Isaac Ampofi, Akyene Tetteh, Eric Neebo Wiah, Sampson Takyi Appiah. Hurst Exponent Analysis on the Ghana Stock Exchange. American Journal of Mathematical and Computer Modelling. Vol. 5, No. 3, 2020, pp. 77-82. doi: 10.11648/j.ajmcm.20200503.13

Received: May 29, 2020; Accepted: June 11, 2020; Published: August 25, 2020

\begin{abstract}
This paper talks about the application of Hurst Index on the Ghana Stock Exchange (GSE). The aim of the paper was to find out, whether GSE daily returns have some autocorrelation (long-term dependency) and multifractality using the Hurst Index analysis. Hurst Index of daily returns of some selected stocks in the period of January 2018 to December 2018 constituting 247 trading days were computed using Rescale Range Method and the Periodogram Method. The findings show that, $91.7 \%$ of the stocks considered possess long-term dependency and only $8.3 \%$ shows multifractality. Besides, the average percentage error of the geometric fractional Brownian motion (GFBM) model was $16.68 \%$ with an efficiency accuracy of $83.32 \%$ whilst that of the geometric Brownian motion (GBM) model percentage error is $20.90 \%$ with an accuracy of $79.10 \%$. This indicates that, the GFBM model yielded better predicting accuracy than GBM in the long-run and par predicting accuracy in the short-run.
\end{abstract}

Keywords: Stock Price, Hurst Exponent, Geometric Brownian Motion, Geometric Fractional Brownian Motion, Ghana Stock Exchange, Drift, Volatility, Ghana Commercial Bank

\section{Introduction}

The Ghana Stock Exchange (GSE) which is one of the youngest and fastest growing Exchange in Africa has seen fewer contribution of literatures on stochastic modelling of its stock prices. Literature from [1], stipulated that, this few literatures on stochastic modelling have made stock prices of the Exchange very difficult to predict. Also, the few literatures all focus on the Geometric Brownian Motion (GBM). This is evident from [1-3].

According to [4], the GBM could not account for the longrun prediction because of its memoryless property. Due to this deficiency, this paper studied the Geometric Fraction Brownian Motion which incorporates the Hurst Index as the catalyst for long-run prediction. The Hurst Index $(0 \leq H \leq 1)$ classifies a time series into three different groups. If $H=0.5$, then the series follow a random walk which means returns are uncorrelated and random. If $0 \leq H<0.5$, then the time series is said to have anti-persistent behavior, which means it is mean reverting. The series is said to have persistent behavior if $0.5<H \leq 1$.
From [15], the Hurst Index gives a clear identity of the stock whether it will predict better on the GBM or GFBM. Therefore, this paper estimates the Hurst Index of some selected stocks from the GSE using the Rescaled Range Analysis and the Periodogram Method and test which stocks predict better using GBM and GFBM.

\section{Literature Review}

In finance, the Black-Scholes option pricing model consist of a risky asset, stocks $S(t)$ and a risk-free asset, a bond. The asset can be modelled as a stochastic process which follows a Geometric Fractional Brownian Motion (GFBM) and define by a stochastic differential equation

$$
d S(t)=\mu S(t) d t+\sigma d B^{H}(t)
$$

In GFBM model, stock returns are dependent of each other, meaning price changes exhibit some autocorrelation called long-range dependency. This long-range dependency has been investigated by many researchers in other stock markets. [5], investigated the Brazilian equity market and found 
persistency, more importantly the results suggest that, the Hurst Exponent is time varying even after adjusting shortrange dependency. [6, 7], show evidence of long memory on the European Options through a time-dependent volatility. [8] agued from Efficient Market Hypothesis (EMH) on Australian market that, if a stock time series has a high Hurst Exponent, then the stock will be less risky and there will be less noise in the data set.

The EMH which originates from Fama's PhD dissertation in the 1960s, states that, at any given time all information about a security has been accounted for in the current price of that security. The logical results of this hypothesis are that, security analyst is unable to predict stocks that will produce excess returns but rather conform to the market price. According to [9], the indecisiveness of the Efficient Market Hypothesis has given room for more studies to be conducted.

The systematic work from [10] has shown that, most studies have empirically proven that, the returns on the Ghana Stock Exchange are predictable. Most of these studies which were on the Efficient Market Hypothesis (EMH) concluded that, the Ghana Stock Exchange (GSE) was weakform inefficient. This makes the Market highly predictable and investors can make abnormal returns by studying past prices on the Market. [11] studied the returns on the GSE between 1993 to 1995 using serial correlation and found that, the market was not weak-form efficient. Also, [12] examined the Efficient Market Hypothesis on the Ghana Stock Exchange and other Stock Exchanges in Africa such as South Africa, Nigeria and Zimbabwe using PACF and White Test. The finding from the study was that, the Ghana Stock Exchange was weak-form inefficient.

[13] Analyzed the South African, Ghanaian, Mauritanian and the Egyptian market using the Run Test, Multiple Variance Ratio (MVR) Test and ARIMA and found all market to be weak-form efficient. The inconsistencies of the EMH on the GSE by literatures cited above makes the market vulnerable and highly predictable but one parameter (Hurst Exponent) that has been used in other Exchanges to confirm the Efficient Market Hypothesis is yet to be tested on the Ghana Stock Exchange, therefore this is the aim of this paper.

\section{Methodology}

The Rescaled range analysis is a statistical measure of the variability of a series that depends on time which was introduce by British Hydrologist Hurst and later explained by [14]. The purpose of the model was to provide an assessment of how the apparent variability of a series changes with the magnitude of the time-period being considered. It is estimated as follows;

If $X$ is the closing price of the stock, then at time $t$, the stock price series is given by

$$
X=X_{1}, X_{2}, \ldots, X_{t}
$$

The expectation of the series in Equation (1) is calculated as

$$
m=\frac{1}{n} \sum_{i=1}^{n} X_{i}
$$

where $m$ is the mean, $n$ is the sample size and $X_{i}$ is the stock price process. A mean adjusted series is created see Equation (4).

$$
Y_{t}=X_{t}-m, \forall t=1,2, \ldots, n
$$

Cumulative deviate series is also created and can be found in Equation (5)

$$
Z_{t}=\sum_{i=1}^{t} Y_{i}, \forall t=1,2, \ldots, n
$$

The range of the series is computed as;

$$
R(n)=\max \left(Z_{1}, Z_{2}, \ldots, Z_{n}\right)-\min \left(Z_{1}, Z_{2}, \ldots, Z_{n}\right)
$$

and the standard deviation is also computed as;

$$
S(n)=\sqrt{\frac{1}{2} \sum_{i=1}^{n}\left(X_{i}-m\right)^{2}}
$$

Finally, the rescaled range $(\mathrm{Rr})$ is computed and it is shown in Equation (8)

$$
R r=\frac{R(n)}{S(n)}
$$

The Hurst Index is estimated by fitting the power law $E\left[\frac{R(n)}{S(n)}\right]=C n^{H}$ to the data. This can be done by plotting $\log \left[\frac{R(n)}{S(n)}\right]$ as a function of $\log n$, and fitting a straight line; the slope of the fitted line is the Hurst Index $(\mathrm{H})$.

[16] Apply fractional Brownian motion $B_{H}(t)$ to replace the classical Brownian motion $B(t)$ and further incorporate the existence of long memory in financial market. The suggested model is as follows:

$$
d X(t)=\mu X(t) d t+\sigma d B_{H}(t), H \in\left(\frac{1}{2}, 1\right)
$$

Analytical Solution of GFBM

Since $H \neq \frac{1}{2}, B_{H}(t)$ is not a semi martingale; thus, the general theory of stochastic calculus cannot be applied on $B_{H}(t)$ instead, Wick calculus is used. Now, we assume the initial condition $X(0)=X_{0}$. The stochastic differential equation in Equation (9) can be written as:

$$
\frac{d X(t)}{d t}=\mu X(t)+\sigma X(t) \frac{d B_{H}(t)}{d t}
$$

applying Wick calculus, Equation (10) can be rewritten in 
$(S)_{H}^{*}$ as:

$$
\frac{d X(t)}{d t}=\mu X(t)+\sigma X(t) W_{H}(t)
$$

or

$$
\frac{d X(t)}{d t}=\left(\mu+\sigma W_{H}(t)\right) X(t)
$$

integrating both sides of Equation (11) gives:

$$
X(t)=X_{0} \exp \left(\mu t+\sigma \int_{0}^{t} W_{H}(u) d u\right)
$$

given $\frac{d}{d t} B_{H}(t)=W_{H}(t)$ in $(S)_{H}^{*}$, Equation (12) can be written as:

$$
X(t)=X_{0} \exp \left(\mu t+\sigma B_{H}(t)\right)
$$

now, we introduce Definition 1 and Definition 2 from [13] to make further deduction.

Definition 1. If $Y: \mathbb{R} \rightarrow(S)_{H}^{*}$ is a given function provided that $Y(t) W_{H}(t)$ is integrable in $(S)_{H}^{*}$, then we can define the fractional Wick-Ito integral of a function as:

$$
\begin{gathered}
\int_{\mathbb{R}} Y(t) d B_{H}=\int_{\mathbb{R}} Y(t) W_{H}(t) d t \\
\int_{0}^{t} B_{H}(S) d B_{H}(S)=\frac{1}{2} B_{H}^{2}(t)-\frac{1}{2} t^{2 H}
\end{gathered}
$$

Definition 2. Let $f \in L_{H}^{2}(\mathbb{R})$, then;

$$
\exp (\langle w, f\rangle)=\xi(f)=\exp \left(\int f d B_{H}-\frac{1}{2}\|f\|_{H}^{2}\right)
$$

applying Definition 2 and Equation (15), Equation (12) can be represented as:

$$
\begin{aligned}
& X(t)=X_{0} \exp \left(\mu t+\sigma B_{H}(t)-\frac{1}{2} \sigma^{2} t^{2 H}\right) \\
& X(t)=X_{0} \exp \left(\mu-\frac{\sigma^{2}}{2}\right) t^{2 H}+\sqrt{\sigma^{2}} \xi(t) \sqrt{t^{2 H}}
\end{aligned}
$$

substituting $\xi(t) \sim N(0,1)$, we get;

$$
X(t)=X_{0} \exp \left(\mu-\frac{\sigma^{2}}{2}\right) t^{2 H}+\sqrt{\sigma^{2}} N(0,1) \sqrt{t^{2 H}}
$$

Equation (18) is the analytical solution of the GFBM based on Wick calculus and it is used for predicting asset price in financial market.

\section{Results and Discussion}

To access whether the log returns of the selected stocks are valid for normal distribution, statistical test was performed using the Shapiro-Wilk test, Kurtosis and Skewness which have consistently been proven valid by many literatures. Table 1 shows the normality test performed on the log returns.

Table 1. Descriptive statistic of the log returns.

\begin{tabular}{lllll}
\hline Stocks & Mean & Shapiro-Wilk & Kurtosis & Skewness \\
\hline ACCESS & 0.0136 & $0.0048 * * *$ & 8.1267 & -0.2776 \\
ADB & 0.0001 & $0.0000 * * *$ & 114.8568 & 10.2958 \\
BOPP & 0.0056 & $0.0041^{* * *}$ & 89.2052 & -0.5079 \\
EGH & 0.0125 & $0.0060 * * *$ & 69.1591 & 0.0761 \\
EGL & 0.0127 & $0.0092^{* * *}$ & 114.0185 & 2.0148 \\
GCB & 0.0378 & $0.0362 * * *$ & 113.9066 & 0.0237 \\
GGBL & 0.0028 & $0.0012 * * *$ & 25.1948 & 0.3152 \\
GOIL & 0.0101 & $0.0040 * * *$ & 46.0792 & 0.1858 \\
SCB & 0.0109 & $0.0061 * * *$ & 79.7195 & -0.1133 \\
SOGEGH & 0.0142 & $0.0055^{* * *}$ & 65.2453 & 1.7717 \\
TOTAL & -0.0002 & $0.0059^{* * *}$ & 57.3257 & -0.5532 \\
UNIL & 0.0034 & $0.0016 * * *$ & 45.4749 & 3.1780 \\
\hline
\end{tabular}

To estimate the Hurst exponent from the selected stocks on the GSE, the rescaled range analysis method was adopted. The results are shown in Figure 1 and Table 2.

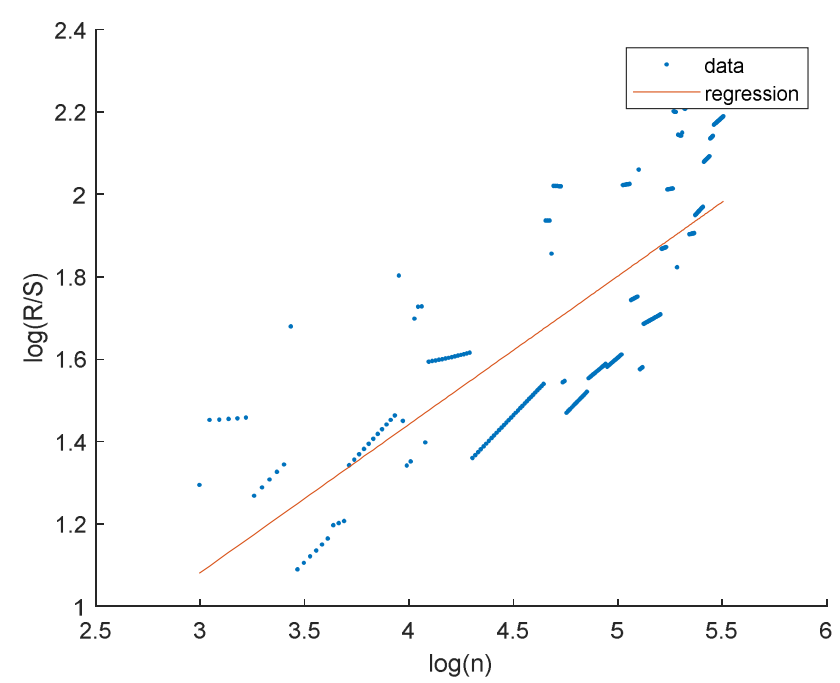

Figure 1. Hurst Exponent Estimation.

Table 2. Hurst Exponent Estimate.

\begin{tabular}{llll}
\hline Stocks & $\begin{array}{l}\text { Rescaled range } \\
\text { Analysis }\end{array}$ & $\begin{array}{l}\text { Periodogram } \\
\text { Method }\end{array}$ & P-Value \\
\hline ACCESS & 0.3597 & 0.2593 & 0.1070 \\
ADB & 0.3933 & 0.3719 & 0.5052 \\
BOPP & 0.6752 & 0.6389 & $0.0000^{* * *}$ \\
EGH & 0.5856 & 0.5877 & $0.0000^{* * *}$ \\
EGL & 0.4739 & 0.1975 & $0.0000^{* * *}$ \\
GCB & 0.4159 & 0.1405 & $0.0000^{* * *}$ \\
GGLB & 0.3907 & 0.3779 & $0.0000^{* * *}$ \\
GOIL & 0.8002 & 0.7989 & $0.0000^{* * *}$ \\
SCB & 0.7519 & 0.6414 & $0.0000^{* * *}$ \\
SOGEGH & 0.7540 & 0.6732 & $0.0000^{* * *}$ \\
TOTAL & 0.7111 & 0.6331 & $0.0000^{* * *}$ \\
UNIL & 0.3593 & 0.6220 & $0.0004 * * *$ \\
\hline
\end{tabular}


From Table 2, ACCESS and ADB were the negatively autocorrelated stocks with rescaled range value of 0.3597 and 0.3933 respectively. The value of the periodogram is also in line with the rescaled range value with ACCESS having a periodogram value of 0.2593 and 0.3719 for ADB.

The Hurst value from both the periodogram and the rescaled range analysis for ACCESS and ADB were confirmed by the heteroscedasticity test with p-value of $10.7 \%$ and $50.5 \%$ for ACCESS and ADB respectively. This means that at 5\% significant level, inferences from past data of these stocks will be relatively inaccurate. From Table 2, BOPP, EGH, SCB, SOGEGH and TOTAL are highly persistent with Hurst exponent of $0.6752,0.5856,0.7519,0.7540$ and 0.7111 and also $0.6389,0.5877,0.6414,0.6732$ and 0.6331 for rescaled range and periodogram respectively. The test statistics was $0 \%$ which was significant at 5\% significance level. But the most persistent or positively autocorrelated stock was the GOIL stock with rescaled range value of 0.8002 and a periodogram value of 0.7989 . Stocks with high Hurst exponent can be predicted using their past values.

In summary from Table 2, out of the 12 equities considered, only 3 of them (ACCESS, ADB and UNIL) did not exhibit the long memory hypothesis, the remaining (BOPP, EGH, EGL, GCB, GGLB, GOIL, SCB, SOGEGH and TOTAL) all have long memory characteristics. This means that $75 \%$ of the stocks considered can be modelled using the geometric fractional Brownian motion.

After the model specification, simulation was done for the different values of Hurst exponent in the short-run and the long-run and the results are shown in Figure 2 to Figure 4.

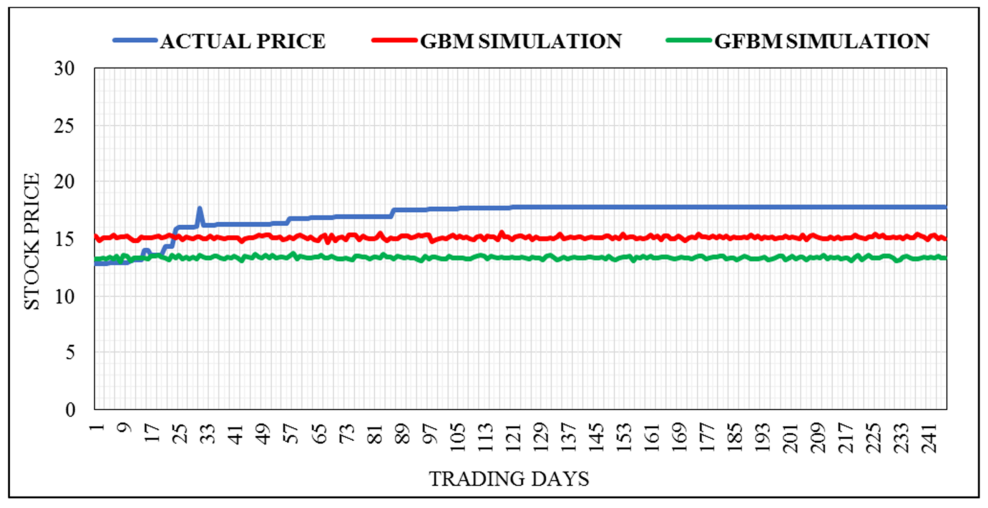

Figure 2. Short-Run and Long-Run Simulation of UNIL Stock with $H<0.5$.

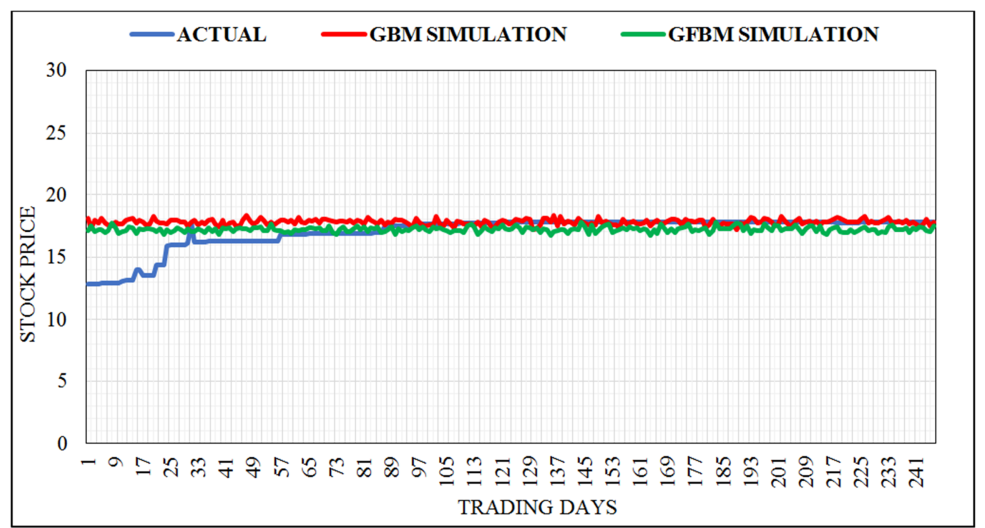

Figure 3. Short-Run and Long-Run Simulation of UNIL Stock for H=0.5.

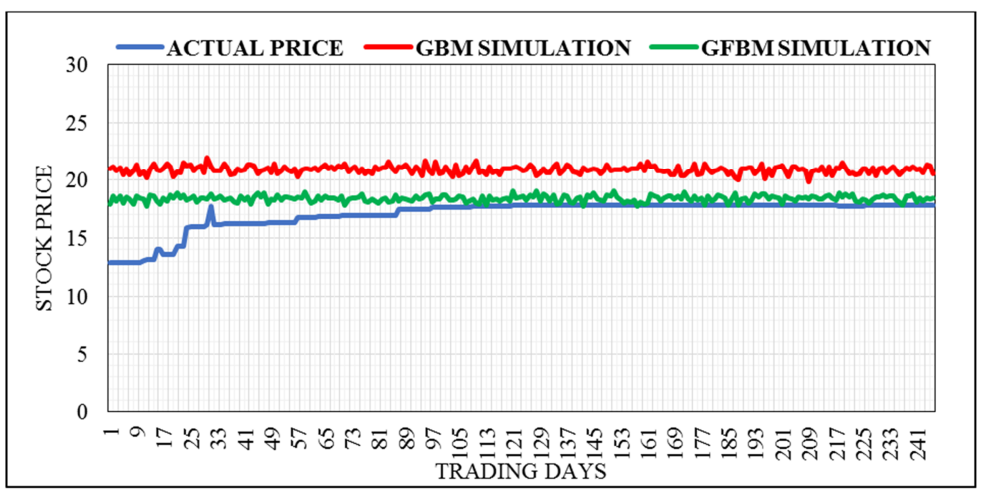

Figure 4. Short-Run and Long-Run Simulation of UNIL Stock for H>0.5. 
From Figure 2, it can be seen that, the short-run and the long-run simulation were made for Hurst exponent less than 0.5 and under both cases, the GBM model simulated values were closer to the actual than it was to the GFBM model. Figure 2 is the simulation for only the UNIL stock and the simulation were performed for the remaining stocks considered and the results are presented in Table 3 and Table 4.

In Figure 3, simulations for the short-run and the long-run were made at Hurst exponent of 0.5. From the model development, it was deduced that at $\mathrm{H}=0.5$, the GBM model and the GFBM model are the same. This argument holds for the simulation since the predictability of the two models were the same.

For Hurst exponent greater than 0.5, the stock price is persistent with time and for that, the GBM model decays exponentially to zero. Meanwhile the GFBM model at this Hurst exponent (0.5) give the best prediction. This is evident in Figure 4, where the simulation of the GFBM model predicts far better than the GBM model. The Figure 4 is the prediction for only the UNIL stock, the remaining equities were also simulated and the results are shown in Table 3 and Table 4.

Table 3. Short-Run Predicted Stock Prices.

\begin{tabular}{llllll}
\hline \multirow{2}{*}{ Stock } & \multirow{2}{*}{ Actual Stock Price (GHS) } & \multicolumn{2}{l}{ Predicted Stock Price (GHS) } & \multicolumn{2}{l}{ Percentage Error } \\
\cline { 3 - 6 } & & GBM Model & GFBM Model & GBM Model & GFBM Model \\
\hline UNIL & 17.0354 & 15.1118 & 13.3704 & 13.18 & 21.34 \\
ACCESS & 3.7637 & 3.7985 & 3.9847 & 6.51 & 7.78 \\
SCB & 27.7921 & 23.0129 & 24.6936 & 18.62 & 15.96 \\
ADB & 5.9474 & 5.8951 & 5.8534 & 0.9 & 1.58 \\
BOPP & 6.6162 & 5.5936 & 5.9913 & 17.66 & 14.87 \\
EGH & 9.3365 & 7.5656 & 7.5838 & 17.88 & 17.91 \\
EGL & 3.514 & 2.9006 & 3.5037 & 18.49 & 20.93 \\
GOIL & 3.7083 & 2.9681 & 2.7983 & 19.05 & 22.72 \\
GGBL & 2.4206 & 2.1181 & 2.0733 & 12.22 & 13.99 \\
TOTAL & 4.6767 & 3.5525 & 3.6187 & 22.85 & 21.47 \\
GCB & 5.6555 & 5.0377 & 5.1133 & 29.34 & 29.39 \\
SOGEGH & 1.4153 & 0.7854 & 0.8122 & 37.88 & 36.17 \\
Average & & & & 17.88 & 18.68 \\
\hline
\end{tabular}

Table 4. Long-Run Predicted Stock Prices.

\begin{tabular}{llllll}
\hline \multirow{2}{*}{ Stock } & \multirow{2}{*}{ Actual Stock Price (GHS) } & \multicolumn{2}{l}{ Predicted Stock Price (GHS) } & \multicolumn{2}{l}{ Percentage Error } \\
\cline { 3 - 6 } & & GBM Model & GFBM Model & GBM Model & GFBM Model \\
\hline UNIL & 17.0354 & 20.9281 & 18.4112 & 23.74 & 9.85 \\
ACCESS & 3.7637 & 3.3128 & 3.5136 & 12.54 & 9.82 \\
SCB & 27.7921 & 19.2346 & 20.7699 & 28.85 & 13.97 \\
ADB & 5.9474 & 6.0059 & 5.9619 & 0.98 & 0.24 \\
BOPP & 6.6162 & 4.6502 & 4.9970 & 28.48 & 15.46 \\
EGH & 9.3365 & 7.4501 & 7.4844 & 19.15 & 18.91 \\
EGL & 3.5140 & 1.7534 & 2.1479 & 47.42 & 15.76 \\
GOIL & 3.7083 & 3.4206 & 3.2261 & 15.68 & 16.32 \\
GGBL & 2.4206 & 2.2456 & 2.1935 & 8.30 & 9.55 \\
TOTAL & 4.6767 & 3.4290 & 3.4769 & 25.26 & 24.31 \\
GCB & 5.6555 & 4.6381 & 4.8659 & 33.82 & 31.22 \\
SOGEGH & 1.4153 & 0.7191 & 0.7421 & 42.68 & 10.77 \\
Average & & & & 23.91 & 14.68 \\
\hline
\end{tabular}

The Mean Absolute Percentage Error (MAPE) of the GBM and GFBM models were estimated. The average MAPE of the GBM model was $17.88 \%$ which indicates a good prediction and the average MAPE the GFBM model was $18.68 \%$ which also indicate a good prediction. These predictions were all in the short-run, but in the long-run, the average MAPE of GFBM was $14.68 \%$ indicating a good prediction whilst the GBM was $23.91 \%$ indicating a moderate prediction.

Comparing the predictability of the GBM and GFBM models, it can be seen from Table 3 and Table 4 that, in the short-run and long-run the GFBM model gave a good prediction whilst the GBM was not good in both cases.

\section{Conclusions}

The research was conducted purposely to develop a model that will take into account the Hurst exponent to model stock price in Ghana. From the findings of the research, the following conclusions were drawn in relation to the objective of the study.

A stochastic differential equation model that takes into account the Hurst exponent using Wick calculus and Ito lemma has been established and this is represented as 
$X(t)=X_{0} \exp \left(\mu-\frac{\sigma^{2}}{2}\right) t^{2 H}+\sqrt{\sigma^{2}} N(0,1) \sqrt{t^{2 H}}$, where $H$ is the Hurst exponent.

Finally, in order to validate the proposed model, numerical simulations of the model were carried out base on the time constraint (long-run and short-run) and was used to compare other theoretical models. From Table 3 and Table 4, the average mean absolute percentage error of the predictability of the GFBM model was $16.68 \%$ which indicates a good prediction from the scale of judgement while the GBM model average error was $20.90 \%$ indicating moderate prediction. Therefore, the GFBM model predicts stock prices better than the GBM model on the Ghana Stock Exchange.

\section{References}

[1] Antwi, O. (2017) Stochastic Modelling of Stock Price Behaviour on Ghana Stock Exchange. International Journal of Systems Science and Applied Mathematics, 2 (6), p. 116.

[2] Damptey, K. N. O. (2017), Rethinking the role of indigenous governance practices in contemporary governance in Africa, the case of Ghana.

[3] Quayesam, D. L. (2016), Stochastic Modelling of Stock Prices on The Ghana Stock Exchange (Doctoral dissertation, University of Ghana).

[4] Zili, M. (2006), on the mixed fractional Brownian motion. International Journal of Stochastic Analysis.

[5] Bekaert, G., Harvey, C. R. and Lundblad, C. T. (2003), Equity market liberalization in emerging markets. Journal of Financial Research, 26, (3), pp. 275-299.

[6] Jamdee, S. and Los, C. A. (2005) Long memory options: Valuation. Available at SSRN 588862.

[7] Jamdee, S. and Los, C. A., (2007), Long memory options: LM evidence and simulations, Research in International Business and Finance, 21, (2), pp. 260-280.

[8] Fortune, P., (1991), Stock market efficiency: an autopsy. New England Economic Review, pp. 17-40.

[9] Gupta, K. and Singh, B., (2006), Random walk and indian equity futures market. In Indian Institute of Capital Markets 9th Capital Markets Conference Paper.

[10] Afego, P. N. (2015), Market efficiency in developing African stock markets: what do we know? The Journal of Developing Areas, pp. 243-266.

[11] Osei, K. A., (2006), Macroeconomic factors and the Ghana stock market, African finance journal, 8, (1), pp. 26-38.

[12] Ntim, C. G., Opong, K. K. and Danbolt, J., (2007), An emperical re-examination of the weak form efficient markets hypothesis of the Ghana Stock Market using variance-ratios tests, African Finance Journal, 9, (2), pp. 1-25.

[13] Adobaw, I., (2014), TESTING THE WEAK FORM MARKET EFFICIENCY OF SELECTED AFRICAN STOCK MARKETS (Doctoral dissertation, Department of Economics of the Faculty of Social Sciences, University of Cape Coast).

[14] Boes, D. C. and Salas, J. D., (1978), Nonstationarity of the mean and the Hurst phenomenon, Water Resources Research, 14, (1), pp. 135-143.

[15] Gursakal, N., Aydin, Z. B., Gursakal, S. and Tuzunturk, S., (2009), Hurst exponent analysis in Turkish stock market. International Journal of Sustainable Economy, 1, (3), pp. 255269.

[16] Mishura, I. S., Mishura, I. S., Misura, J. S., Mishura, Y. and Misura, U. S., (2008), Stochastic calculus for fractional Brownian motion and related processes, Springer Science and Business Media.

[17] Biagini, F., Hu, Y., Oksendal, B. and Zhang T., (2008), Stochastic calculus for fractional Brownian motion and applications, Springer Science and Business Media. 